\title{
Plaisirs de lire et de regarder : de la littérature à la peinture. La théorie de la relation esthétique à l'épreuve du cas Dubuffet
}

\author{
Alain Trouvé* \\ CRIMEL, Université de Reims
}

Résumé : L’activité de lecture, constitutive de la relation littéraire, ne s'applique que partiellement à un tableau qui offre à l'œil un pôle confusionnel indéchiffrable par les signes de la langue. Le Cabinet logologique de Dubuffet amène le visiteur à faire l'expérience de ce rêve impossible : un nouveau logos susceptible de déchiffrer l'indicible. Le Paladin et la Paladine figurés sur les portes d'accès interrompent la continuité de la fresque recouvrant les murs de la pièce et nous rappellent que la littérature et l'art s'apprécient dans une relation esthétique fondée sur l’altérité et un espace socialisé par le langage.

Mots-clés : littérature, peinture, théorie, logos, (dis)continuité, altérité, inconscient esthétique, arrière-texte

Abstract: Reading activity, which is a constituent part of the literary relationship, is only partially applicable to a painting that offers the eye a confusing pole that cannot be deciphered by the signs of language. Dubuffet's Logological Cabinet invites visitors to experience this impossible dream : a new logos capable of deciphering the inexpressible. The Paladin and the Paladine depicted on the entrance doors break the continuity of the fresco covering the walls of the room and remind us that literature and art are appreciated in an aesthetic relationship based on otherness and a space socialized by language.

Keywords: Littérature, painting, theory, logos, (dis)continuity, otherness, aesthetic unconcious, arrière-texte 
Malgré sa simplicité apparente, l'expression «plaisir de lire » ne se laisse pas définir sans exposer l'interprète à certains abus de langage. Nous nous proposons de dissiper à ce sujet une double confusion.

S’agissant de littérature et d'art, le domaine qui nous intéresse ici, l'assimilation naguère très en vogue du plaisir littéraire à la jouissance demeure problématique. Ce qui sépare les deux notions n'est pas seulement affaire de degré, la jouissance supposant un investissement érotique d'une intensité supérieure, mais de relation: au plaisir littéraire ou artistique, partageable par des mots, s'oppose durablement la jouissance, solitaire.'

Une autre confusion fréquente étend l'acte de lire à d'autres arts et notamment à la peinture. En distinguant « plaisir de lire » et «plaisir de regarder » nous signalons ce fait trop souvent oublié : l'expression « lire un tableau » n'est recevable que par métaphore et jusqu'à un certain point.

Il s'agira en somme de situer ces notions - «plaisir », « lire » - au sein de la relation artistique dans sa dimension esthétique, alliant l'intellect (verbal) et le sensible (non verbal), ce qui amènera à revisiter ou proposer quelques autres notions, de l'inconscient esthétique à l'arrière-texte.

L'investigation sera conduite en deux temps: le cadrage théorique du réseau notionnel impliqué dans le plaisir de lire ouvrira sur l'exploration d'une œuvre singulière, celle de Jean Dubuffet, peintre et plasticien, littérateur et penseur de l'art, figure de la dernière avant-garde du $X X^{e}$ siècle, qui joua à sa manière sur les deux plans de la lecture et du regard.

\section{La théorie littéraire : un ensemble articulé de notions}

Comme tout langage de communication, la théorie littéraire, repose sur des catégories partageables, censées transmettre des connaissances relatives à leur objet. C'est par le signifié dénoté des mots (proche selon Saussure du concept), que le langage permet l'échange avec autrui en subsumant sous le général la diversité foisonnante de l'expérience sensible. Il est à noter que Saussure écarta finalement le projet de nommer concept ce qu'il désigna dès le Cours de linguistique générale et de façon plus souple comme signifié:

Nous appelons signe la combinaison du concept et de l'image acoustique : mais dans l'usage courant ce terme désigne généralement l'image acoustique seule, par exemple un mot (arbor, etc.). On oublie que si arbor est appelé signe, ce n'est qu'en tant qu'il porte le concept «arbre», de telle sorte que l'idée de la partie sensorielle implique celle du total.

Nous proposons de conserver le mot signe pour désigner le total, et de remplacer concept et image acoustique respectivement par signifié et signifiant [...] (F. de Saussure [1916] 1974: 99) 
La réticence saussurienne vis-à-vis du « concept » s'explique peut-être par la résistance de «la partie sensorielle » à l'abstraction. Elle vaut encore pour la théorie littéraire quelle que soit son ambition de généralité. C’est pourquoi on préférera nommer ses catégories notions afin de mieux appréhender la complexité de l'expérience esthétique impliquée dans la relation littéraire ou artistique.

On peut se souvenir ici de la distinction posée naguère par Jean-Marie Schaeffer entre les procédures mentales esthétique et scientifique :

Souvent, lorsqu'on compare l'investigation scientifique ( ou savante ) avec la conduite esthétique, on dit que la première suit un mouvement d'abstraction, alors que dans le cadre de la conduite esthétique l'attention est plutôt associative. [...] Mais l'opposition entre abstraction et association ne suffit pas pour rendre compte de la distinction entre les deux niveaux cognitifs. [...] On dira plutôt que c'est la différence entre activité cognitive horizontale (associative) d'un côté, activité cognitive verticale (généralisante ou particularisante) de l'autre, qui distingue la relation cognitive de niveau I [incluant la conduite esthétique] de la relation de niveau 2 [scientifique]. (1996:165-166)

Concevoir la théorie littéraire comme ensemble de notions revient à transférer sur ce terme une part des propriétés (horizontales et associatives) caractéristiques de ce que Schaeffer nomme "conduite esthétique » et que nous appellerons plutôt "activité esthétique », impliquant une projection subjective redoublée, de l’auteur au lecteur.

Ces remarques liminaires s'appliquent aux notions de littérature et d'esthétique elles-mêmes. Au tournant des XVIII et XIXe siècles, la littérature remplace les BellesLettres quand les productions écrites ne sont plus données comme des modèles à admirer mais comme des performances verbales à évaluer. Jacques Rancière situe l'émergence de ce mot au seuil de l'époque moderne où apparaît " la littérature comme régime historique d'identification de l'art d'écrire » (2007: 17). Cette identification implique le jugement d'autrui.

Le surgissement du mot littérature coïncide avec un autre terme récemment introduit: esthétique. C'est Baumgarten qui forge le néologisme en 1750 à partir de l'adjectif grec æesthetica. Son ouvrage éponyme contribue à la constitution de l'esthétique comme discipline. Il envisage une faculté esthétique propre au sujet humain, qu'il donne à concevoir comme intermédiaire entre la sensation et l'intellect.

Tout écrit à vocation littéraire est la trace d'un acte d'écriture-lecture associé à une évaluation esthétique redoublée, de l'auteur au lecteur. La parole est le terme générique approprié pour désigner cet acte innovant. La parole forge un sens intelligible mais reste adossée à son support sensible, le corps, figuré par le style à l'écrit ou par son équivalent oral, la voix. La parole auctoriale a pour corollaire une contre-pa- 
role lectorale, prenant la forme d'un commentaire oral ou écrit plus ou moins élaboré.

Si la littérature implique le jugement esthétique d'autrui et l'ouverture vers sa contre-parole, on comprend pourquoi le plaisir littéraire ne peut sans abus de langage être nommé jouissance. Barthes avait opposé dans son essai de 1973 le «plaisir du texte » qui « peut être dit » aux «textes de jouissance » qui mettent «le plaisir en pièces, la langue en pièces, la culture en pièces ». La parole du théoricien se faisait ici paradoxale, s'employant à dire ce qui ne peut être dit. "Plaisir/Jouissance: Terminologiquement, cela vacille encore, j'achoppe, j'embrouille» (2002 : 219). De fait, la jouissance qui abolit les frontières entre les sujets supprime la distinction entre le sujet auteur et le sujet lecteur, également fascinés par un Texte transcendant. Dans son article " (Théorie du) texte », rédigé la même année pour l'Encyclopédie Universalis, Barthes emploiera la contestable et amusante formule du «texte textuel » pour désigner cette textualité supérieure, censée abolir les frontières. ${ }^{2}$

Nous proposons plutôt, avec Yves Bonnefoy (2016), d'envisager la relation littéraire comme «échange » verbal, formule à laquelle nous ajoutons le participe « différé », afin de prendre en compte l'écart entre l'écriture-lecture initiale, celle de l'auteur, et la ressaisie ultérieure par d'autres. Soit : " la littérature comme échange verbal différé » (Trouvé 2019). Seul le plaisir, avec sa part de réappropriation verbale dans un énoncé contrapunctique, est partageable. L'horizon de la jouissance est irrémédiablement solitaire.

S’il est bon de ne pas confondre les sujets, il importe de rappeler aussi le statut flottant des identités subjectives, compromis entre une pensée consciente et inconsciente. Les siècles passés ont eu l'intuition d'un rapport entre l'art et ce qui ne se nommait pas encore inconscient. Cette intuition se précise chez les poètes à la fin du fin XIX siècle. Parallèlement à l'émergence de la psychanalyse, l'idée d'un inconscient esthétique prend une place importante dans le discours critique au XXe siècle, infléchissant les pratiques d'écriture des avant-gardes, selon deux vagues. I) L'art est d'abord rapporté à l'inconscient psychique freudien, voire jungien, dans la foulée de Dada et du surréalisme, qui se posent parfois en rivaux de l'analyste. 2) Ce que nous appellerons la deuxième vague de la révolution artistique du vingtième siècle s'intéresse plutôt à un inconscient corporel. On peut citer en ce sens une constellation d'artistes: Bataille, Michaux, Ponge, Alechinsky, Pollock, Dubuffet...

Ce renouvellement nous semble proche des réflexions développées par Jacques Rancière dans L'Inconscient esthétique (200I). L'inconscient freudien, note-t-il, traite le non-sens apparent en symptôme d'une histoire, l'inconscient esthétique correspond à « la voix anonyme de la vie inconsciente et insensée » (Rancière 200I: 57). Cette voix provient du contact de l'artiste avec la matière, de son implication corporelle dans le geste artistique.

Dans un esprit voisin, on peut évoquer aussi les travaux de Laurent Jenny. Son essai La Vie esthétique (2013), dépassant le cadre strict des artefacts artistiques, 
étend la notion d'esthétique à toutes les situations humaines, matrices potentielles d'une activité artistique. Jenny définit le «moment esthétique » comme "structure et jeu de relations » : «il est énergie et refiguration, énergie de refiguration » (2013 : 138). Cette énergie puise sa force dans le rapport du sujet au monde.

Un autre essai du même auteur, récemment publié sous le titre Le Désir de voir (2020), nous semble à son tour élargir la perspective freudienne. Freud avait évoqué une pulsion scopique ou scoptophilie ${ }^{3}$ pour désigner le lien inconscient, y compris en art, entre le désir de voir et la Scène primitive. Comment passe-t-on de la pulsion à l'art ? Pour Freud, la sublimation est l'autre destin de la pulsion, à côté des formes pathologiques ( névrose, psychose, perversion ). Le retour chez Jenny au « désir de voir ", substitué à la "scoptophilie », ne procède pas que d'un refus du jargon théorique. Il nous paraît répondre à la gêne éprouvée face au côté réducteur de la sublimation dans la théorie freudienne. Paul Ricoeur avait déjà signalé ce problème d'une pensée de la création artistique appréhendée seulement dans son rapport à la sexualité (Ricoeur 1965: 166).

Revenons à présent à l'assimilation de l'image picturale au texte, annoncée par l'expression « lire le tableau ». La " traduction en mots » est possible jusqu'à un certain point. L'essai de Michel Butor Les Mots dans la peinture (1969) en a exploré les formes et les limites. Dans cette réflexion sur l'art pictural restreint à la sphère occidentale, le romancier rappelle en quoi une longue tradition nous invite et nous aide à « lire les tableaux » en identifiant, grâce à la légende, la représentation de sujets, d'objets ou de lieux appartenant à un référentiel commun à l'artiste et à celui qui regarde le tableau. L'idée s'applique bien à la peinture historique ou mythologique qui transpose en image une scène déjà vécue ou racontée. L'insertion de mots sur la toile elle-même, comme dans le Marat assassiné de Jacques-Louis David (1793), prolonge encore la conversion du visuel en intellect verbalisé. Mais la peinture moderne met en échec cette prétention à rendre le tableau lisible. Si le tableau de Bruegel, La Chute d'lcare (1558), offre au regard attentif des détails du récit légendaire transmis par les Anciens, la toile de l'américain Jasper Johns, Jubilé (1954), reproduite plus loin, associe à la dissolution de toute forme identifiable la dissémination de signes linguistiques noyés dans la pâte et la couleur qui en effacent la lisibilité. II n'est pas surprenant qu'un des chefs de file du «nouveau roman » se soit montré attentif à cet échec partiel d'une transposition verbale de l'image.

Dans la lecture littéraire existe aussi un pôle de passivité consentie, fortement articulé à des images mentales (fantasmatisation), mais cette passivité entre dans un rapport dialectique avec un pôle d'activité, lié au maniement des signes. Cette interaction permet l'autonomie du sujet lisant vis-à-vis du texte lu. Avec la peinture, en revanche, la fascination est plus grande. "Le tableau est 'un piège à regard' ", dit Lacan, cité par Jean Oury (1989: 121). L'image, objet de perception sensible, excède toujours le sens véhiculé par les mots. Aragon remarque en ce sens dans des Entre- 
tiens sur l'art : «Et je pense que si nous entrons dans un musée - eh bien, puisque c'est notre prétexte d'aujourd'hui, dans le musée de Dresde - c'est précisément qu'il y a de l'irréductible aux mots... » (1957:10).

Nous proposons de voir dans cette remarque le prélude à l'adoption de l'arrière-texte, notion utile pour penser les rapports du sujet à la création artistique sous ses différentes formes. On doit l'arrière-texte à l'écrivaine Elsa Triolet, compagne de l'auteur du Paysan de Paris. Elle recourt à ce néologisme dans La Mise en mots (1969); cet essai fut le premier d'une collection nouvelle, "Les sentiers de la création », réunissant à la demande de l'éditeur Skira les réflexions de différents artistes sur leur art. Aragon salua cette intuition et la reprit à son compte dans sa propre contribution, Je n'ai jamais appris à écrire ou les incipit, publiée la même année (1969). L'arrière-texte vient probablement du fonds de la culture russe d'Elsa Triolet-Kagan, du linguiste, poète et mathématicien Khlebnikov, ainsi que du dramaturge Tchékhov, dont elle fut la traductrice. 1969 marque aussi l'avènement d'une autre notion majeure, l'intertexte, proposée par Julia Kristeva dans son essai Séméiotikè à partir de sa lecture de Mikhail Bakhtine dont elle fut une des introductrices en France. Rapidement, l'arrière-texte, d'abord adopté par quelques écrivains ou commentateurs de l'époque, fut supplanté par l’intertexte dont les mérites ne sont plus à démontrer. Mais la prépondérance de l'intertexte comme outil pour penser les questions de création littéraire eut aussi pour effet plus discutable d'imposer l'idée d'un rapport général et exclusif de texte à texte.

L'intérêt de l'arrière-texte, remis en circulation depuis une décennie, réside dans la prise en charge des dimensions hétérogènes à la langue ou excédentaires vis-à-vis des mots, grâce à son préfixe " arrière ». Dans cette optique, l'arrière-texte serait le corollaire de l'intertexte. Un volume de synthèse à trois mains, L'Arrière-texte Pour repenser le littéraire, a paru en 2013 chez Peter Lang. ${ }^{4}$ Deux des quatre couches de sens de l'arrière-texte collectivement repensé intéressent de près notre propos. L'hétérogénéité qui en constitue le trait commun désigne I) l'irréductibilité de la chose vue à la chose dite, 2) l'inconscient corporel et matériel comme couche de sens.

Dans les deux cas, il n'y a pas conversion totale, mais incidence d'un domaine sur l'autre.

Le poète se rend au Musée non seulement pour essayer de dire ce qu'il voit, mais aussi pour appréhender ce qu'il ne peut pas dire.

\section{Dans les pas de Dubuffet, plasticien et écrivain, jusqu'au point d'orgue du Cabinet logologique... \\ Jean Dubuffet (1901-1985) a marqué son époque comme plasticien anticonfor- miste. Il fut à ses heures écrivain et théoricien de l'art. Il appartient à la deuxième vague des avant-gardes du XXe siècle, après Dada et le surréalisme. Quelques traits peuvent unifier ce qui reste un conglomérat flou et disparate allant de Bataille à}


l'expressionnisme américain ( Pollock, Rauschenberg... ) : la récusation de la coupure nature-culture, l'anti-historicisme, l'insurrection contre la rationalité.

Un autoportrait réalisé en 1966, dans le style antiréaliste de l'Hourloupe, qui domine alors sa production picturale et poétique, ressemble pourtant de façon étrange à une photographie des mêmes années. Parallèlement, on peut retrouver les grandes lignes du parcours de l'artiste dans sa Biographie au pas de course, livre écrit en 1985 et publié à titre posthume en 1995. On y lit l'hésitation de Dubuffet entre le commerce de vins, héritage familial, et l'art. Deux périodes dominées par la gestion de cet héritage ( 1930-1935 et 1937-1942 ) s'intercalent entre une pratique artistique envisagée très tôt, dès 1918, mais qui peine à trouver sa raison d'être par défiance vis-à-vis d'un art jugé trop institutionnel. C'est seulement à partir de 1942 que l'artiste trouve définitivement sa voie et s'y consacre pleinement, liant son nom à celui de l'Art Brut.

L’Art Brut est une expression inventée en 1945 et préférée par Dubuffet au primitivisme, en dépit de similitudes avec cette notion analysée par le critique et essayiste Georges-Henri Luquet dès 1930. Luquet définissait le primitivisme par le refus ou l'absence de dressage culturel, identifiant trois formes antérieures à ce dressage : I) L'art naïf, enfantin ou populaire, 2) L'art des aliénés, 3) L'art des peuples dits primitifs (l'art nègre, notamment).

Mais le primitivisme est entré dans les musées et les histoires de l'art comme catégorie. L'Art Brut en pastichant les catégories du primitivisme, privilégie le côté sauvage, irrécupérable de la création picturale. Ce qui n'empêcha pas Dubuffet à son tour d'être reconnu et célébré à l'échelle mondiale, notamment sur le continent nord-américain. Baptiste Brun a récemment étudié ces contradictions dans son essai, Jean Dubuffet et la besogne de l'Art Brut (2019).

La sauvagerie importée dans le langage verbal a donné lieu à l'invention de jargons plus ou moins intraduisibles. Les écrits en "jargon relatif”, ${ }^{5}$ de Ler dla campane (1943) à la Botte à nique (1973), opèrent dans l'idiome commun une double transformation. Ils allient une transcription phonétique aberrante à partir d'une audition privée de l'identification morphologique des mots et une transgression des codes du langage par non-respect de la syntaxe et affirmation provocante d'un niveau de langue populaire ou argotique. Une fois reconnus ces principes d'invention, il est possible de procéder à une sorte de traduction en français courant. Cette transposition se révèle assez décevante. Labonfam abeber [La bonne fermme à Bébert] (1950) affiche une misogynie que sauve seulement son caractère caricatural entaché d'autodérision. La Botte à nique (1973) fut écrit, comme les volumes de Triolet, Aragon et Butor précédemment cités, pour la collection « Les sentiers de la création » de l'éditeur Skira. ${ }^{6}$ Cependant, on se demande, une fois opérée la transposition en français de communication, si cette espèce de métaphore filée assimilant création artistique et jardinage recèle les profondeurs qu'elle semble dissimuler sous sa désinvolture formelle ou si le propos ne relève pas pour une bonne part du canular. ${ }^{7}$ Le comble de 
la «théorie brute", si la formule a le moindre sens, serait alors la mise à bas de toute prétention théorique.

Dans la transgression des codes linguistiques, Dubuffet est allé plus loin, jusqu'à ce qu'il a lui-même appelé « jargon absolu ». Il écrit dans une lettre datée de 1962 qu'un texte en jargon absolu est un « texte composé de mots inventés et dont la signification est problématique » (1967:487).

L'HOURLOUPE, au même moment (1962), se présente comme une suite de rubriques écrites en lettres majuscules. Le texte débute ainsi: " ONUFLE / ODRIYON MARVAQUE ANAT JÈZE CARCANNE L'AMBUQUE JIT L'OFIANT RODINNE LO MARMELE OU ZI OUT PORQUE ARDE HOURLOUPE » (1967: 150). Chaque «mot » évoque plusieurs mots possibles, mais aucun en particulier. L'absence de syntaxe empêche de passer du mot à la phrase. Face à ce qui apparaît comme un simulacre de langage, les réactions peuvent osciller entre l'amusement et l'irritation, surtout si l'on prend en compte l'extension du procédé sur six pages.

Le premier volume de Prospectus (1967) réunit ces textes en jargon et d'autres à caractère théorique, rédigés en langue de communication. On a donc affaire à une pratique linguistique double, dissociant la langue d’invention, poussée jusqu'à l'illisibilité, et celle du commentaire explicatif, recourant à l'idiome commun comme moyen d'échange avec autrui. Quelle est la portée de ce discours théorique? Le titre, Prospectus, n'est pas sans rappeler, peut-être par dérision, les publications à caractère commercial. Mais les quatre volumes finalement publiés sous le titre Prospectus et tous écrits suivants, en deux vagues (1967, 1995), soit près de deux mille pages, montrent l'envergure du projet difficilement réductible à une simple plaisanterie.

Dans ses écrits réflexifs, Dubuffet énonce le paradoxe d'une théorie anti-théorique caractérisée par le refus de définir. Une note datée de janvier 85 et placée en tête du volume III des Prospectus précise : "C'est une erreur de croire qu'on peut énoncer de ses idées des formulations bien fidèles. On en représente qu'une face, or elles en ont plusieurs ". Cette déclaration va de pair avec le refus de faire école, sur fond d'individualisme.

Aussi n'est-on pas surpris de l'échec d'une collaboration suivie avec André Breton, un temps entrevue par les deux artistes. Le fondateur du surréalisme qui dirige a la première avant-garde du siècle, dans l'entre-deux guerres, se montra intéressé par l'Art Brut et se rapprocha de Dubuffet en qui il vit, au retour de son exil aux USA, le moyen de relancer son mouvement. Une collaboration temporaire entre les deux hommes s'établit autour de «l'art des fous » en 1948-1949. On connaît l'intérêt porté de longue date par les surréalistes à la folie comme voie d'accès à un inconscient réprimé par la Société. Breton rédigea un article intitulé "L'Art des fous » qui devait prendre place dans le projet d'un Almanach de l'Art Brut réalisé avec Dubuffet. Mais ce projet ne se concrétisa pas pour des raisons complexes de divers ordres. Au plan psychologique, l'individualiste Dubuffet était réfractaire à l'esprit de groupe qui pré- 
valut chez les surréalistes, a fortiori à la tutelle possible d'un chef en qui Breton continua de se penser. Leurs philosophies différaient : l'idéalisme surréaliste de Breton est éloigné du matérialisme implicite, perceptible chez Dubuffet. Enfin les pratiques artistiques de chacun ne renvoient pas au même inconscient esthétique. Breton rêve dans L’Amour fou (1937) d'une écriture poétique franchissant les barrières psychiques entre le moi conscient du créateur et son inconscient. Dubuffet, sans exclure la dimension psychique, est plus tourné vers un inconscient matériel. Quelle qu'en soit la version, psychique ou matérielle, l'artiste, selon lui, le donne à apercevoir dans toutes ses dimensions sans prétendre l'interpréter.

Revenons, pour appréhender cette pratique originale, au cycle de L'Hourloupe qui donna lieu, durant douze années, à une double production d'écrivain et de plasticien. La Biographie au pas de course évoque ce mot inventé :

Le mot « Hourloupe » était le titre d'un petit livre publié récemment et dans lequel figuraient, avec un texte en jargon, des reproductions de dessins aux stylos-billes rouge et bleu. Je l'associais par assonance à « hurler », « hululer », « loup », « Riquet à la Houppe » et le titre «Le Horla» de Maupassant inspiré d'égarement mental. (1995, IV : 5IO)

«Hourloupe » relève d'un travail poétique sur la langue, à la manière de Lewis Carroll. Le mot-valise est partiellement intraduisible : son signifiant excédera toujours son signifié, ce qui n'empêche pas de proposer des équivalences partielles. La référence au Horla de Maupassant signale l'accointance de l'invention verbale avec le récit fantastique et son envers fantasmatique. «Un plongeon dans le fantasme, dans un fantomatique univers parallèle ", écrit encore Dubuffet (idem : 510). En un sens L'Hourloupe ouvre bien sur l'inconscient psychique.

Mais ce qui fait le caractère plus rare de l'entreprise est son caractère double, verbal et pictural, présent dès l'édition d'origine qui associait du texte et des dessins d'abord griffonnés au stylo à bille, puis spectaculairement amplifiés:

[L'Hourloupe] allait se développer dans les années suivantes, en nombreuses peintures, dont certaines prirent de grandes dimensions, puis en sculptures peintes, qui s'agrandirent aussi jusqu'à devenir pour finir des monuments et édifices. (idem : 509)

À Périgny-sur-Yerres (94), non loin de Paris, a été ainsi édifiée entre 1971 et 1976, la Closerie Falbala, avec en son sein, La Villa Falbala, sorte de musée-tombeau de l'artiste, à lui seul dédié. Les portes de la Villa Falbala ouvrent sur une antichambre puis sur le «Cabinet logologique », qui condense peut-être toute la méditation de Dubuffet sur son art.

Logologique : le néologisme dérive du Grec; il s’agit, comme l'explique une lettre de septembre 1969 à Arnold Glimcher, d’inventer un « nouveau logos »: 
une écriture uniforme, [...], à la faveur de quoi s’abolissent toutes les particularisations, toutes les catégories [...] départicularisante, indifféremment appliquée à tous les objets. (Prospectus, III : 354)

Autrement dit, le projet s'attaque au fondement de la pensée occidentale depuis plus de deux millénaires : rien que cela... Le logos a pour les Grecs le double sens de langue et de rationalité. Se débarrasse-t-on si facilement de la langue et de sa dimension rationalisante?

L'Hourloupe, quoi qu'il en soit, porte à son acmé la question du langage et de la figuration. Un an plus tard, Dubuffet y revient :

Je crois qu'on peut trouver dans tous mes travaux constamment, et déjà dès leur commencement en 1942 une alternance d'humeur qui me porte tantôt à mettre l'accent - un accent qu'on pourrait dire expressionniste, non exempt d'outrance caricaturale - sur la spécificité des objets figurés, tantôt au contraire à ne plus donner à cette spécificité qu'une considération réduite, outrancièrement réduite, supposant un monde où tout procède d'un sang unique et où les objets n'ont plus guère d'individuation propre, ne sont plus guère identifiables. (Note de 1970, Prospectus, III : 313)

Oscillation remarquable. L'autre point important dans cette note est le lien qu'elle permet d'établir entre objet identifiable et catégorie verbale (mot). Ce lien était à vrai dire préfiguré par l'application de l'adjectif "logologique » à une entreprise picturale et par l'emploi, pour la définir, du terme «écriture ». Sauf à jouer avec l'illisibilité de cette écriture d'un genre nouveau, nous voici presque revenus au projet, critiqué plus haut, d'unifier le texte et l'image.

Le réel, y compris quand il est perçu par nos sens, ne serait-il qu'illusion de langue susceptible d'être dissipée par une entreprise radicale ? Dans une « lettre [...] aux visiteurs de l'exposition de ses travaux au Havre », un inédit de 1976, Dubuffet réaffirme cette ambition :

On attend d'un peintre qu'il ouvre des voies neuves aux regards que nous portons sur notre quotidien entourage. [...] Or il faut prendre conscience que ce que nous prenons pour le réel et qui nous apparaît fortement comme tel (comme seul tel) n'est rien de plus qu'une arbitraire interprétation des choses à laquelle pourrait aussi bien être substituée une autre. (Prospectus, III : 333)

L'enjeu de cette recherche est-il une connaissance plus profonde ou ne mène-t-il qu'à une dissolution de la connaissance?

Avant de nous rendre, en pensée ou par l'image, dans ce lieu singulier du cabinet logologique pour tenter d'esquisser une réponse, nous proposons un détour par Henri 
Michaux, lui aussi écrivain et peintre, plus connu, toutefois, à l'inverse de Dubuffet, pour ses écrits poétiques que pour ses dessins à l'encre, néanmoins remarquables.

Les deux hommes se sont rencontrés en 1945 et sont devenus amis. Une correspondance suivie porte la marque de cette appréciation mutuelle fondée notamment sur une commune volonté de casser les codes de base de la figuration, picturale ou verbale. Michaux écrit dans Emergences résurgences ${ }^{9}$ : "je peins pour me défamiliariser» (1972: 9). Toutefois, le texte de cet essai diffère profondément de celui de $L a$ Botte à Nique. Michaux choisit de montrer et de commenter sa production picturale, présentée dans un ordre à peu près chronologique. Ce commentaire écrit dans une langue de communication non transgressive laisse de côté les inventions langagières de sa propre poésie qui joua abondamment avec la déstructuration du signifiant ( voir à ce sujet le célèbre «Grand combat » : "Il l'emparouille et l'endosque contre terre... »). Autrement dit, même s'il ne traite que de sa propre œuvre picturale, le commentaire de Michaux se rapproche de l'essai dans sa dimension théorique; le livre de Dubuffet, pour sa part, prendra place au sein du volume III de ses $\varepsilon_{\text {crits, dans }}$ les «pièces littéraires ", poussant plus loin le franchissement de la frontière entre création transgressive et réflexion théorique susceptible de transmettre un savoir socialisé.

Michaux marque aussi de façon plus nette ce qui pourrait différencier une création verbale et une création picturale. Au début de son essai, il oppose deux dessins datés de 1927 et qui correspondent à une sorte d'incipit de toute son œuvre picturale. Le premier est réalisé d'un seul trait de crayon, ainsi que le note le commentaire d'accompagnement :

Une ligne plutôt que des lignes. Ainsi je commence, me laissant mener par une, une seule, que sans relâcher le crayon de dessus le papier je laisse courir, jusqu'à ce qu'à force d'errer sans se fixer dans cet espace réduit, il y ait obligatoirement arrêt. (p. II)

L'ensemble constitue une forme allongée et vaguement ovoïde. La ligne sinueuse fait retour sur son propre tracé qu'elle recoupe par endroits, elle oscille entre gribouillis et amorces de formes éventuellement identifiables.

Le second dessin répartit sur cinq lignes parallèles des sortes de signes ou de pictogrammes dont les formes d'abord extrêmement embrouillées tendent à se simplifier. Ils constituent pour l'œil et pour l'esprit un horizon potentiel de déchiffrement :

Plus tard, les signes, certains signes. Les signes me disent quelque chose. J'en ferais bien, mais un signe, c'est aussi un signal d'arrêt. (p. 13)

Ce qui différencie ce dessin du précédent est en effet l'opposition de la discon- 
tinuité des pseudo-caractères à la continuité de la ligne. À la question - La peinture est-elle un langage? -, les deux dessins de 1927 apportent d'emblée une réponse double : la peinture est potentiellement, par les signes, le discontinu, un langage ou un simulacre de langage ; par la ligne, le continu, l'inentamé, elle diverge radicalement de tout langage.

Nous allons voir à présent que le Cabinet logologique, rêvant peut-être à un langage de l'art supérieur, récuse cette frontière avant de la rétablir, in extremis. Ouvrons enfin les portes de cet espace pour contempler l'espèce de fresque tapissant les quatre murs d'une pièce vide de dessins réalisés dans le style de L'Hourloupe ; chaque dessin se compose de quasi formes enchevêtrées, contiguës à d'autres dessins, l'ensemble formant pour l'œil une une continuité presque sans faille. Cette fresque indéchiffrable n'est pas sans rappeler les tombeaux égyptiens. Mais pas de Champollion pour en traduire le texte, peut-être seulement fantasmé. A la différence des hiéroglyphes, la fresque du Cabinet n'offre pas à l'œil de signes discontinus. L'artiste, selon le commentaire proposé au visiteur, venait s'asseoir et méditer de longues heures, au centre de cet espace.

Il nous faut enfin prendre en considération la porte à deux battants qui ouvre et referme l'accès à cette pièce ${ }^{10}$ :

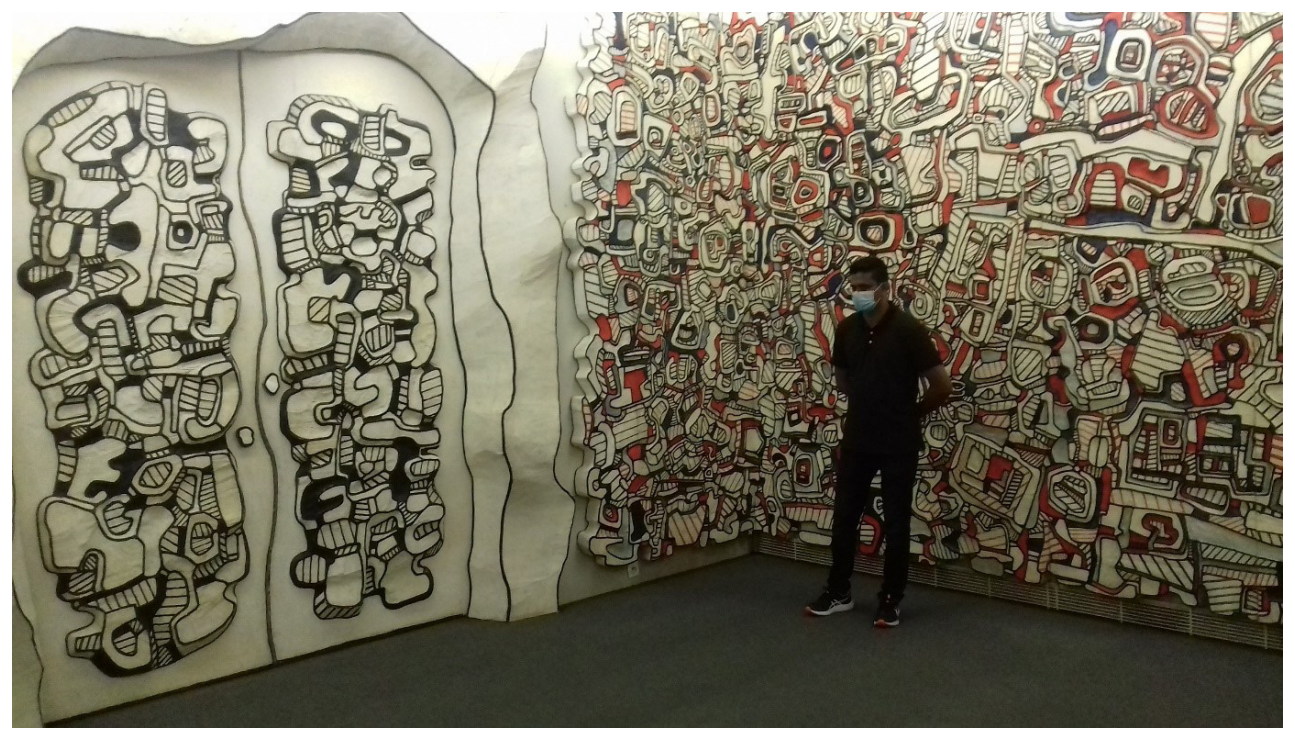

Sur les portes on voit deux figures distinctes, nommées par l'artiste, le Paladin et la Paladine. Selon le jeu proposé au visiteur par les guides assurant la visite, il s'agirait de retrouver qui est qui. Peu importe, sans doute, la réponse aléatoire à cette question. Ce qui compte est qu'elle soit posée, qui plus est, à des tiers. Autrement dit, si la fresque en continu correspond à la faillite du logos des Grecs, tout se passe 
comme si la séparation des figures et la distinction supposée entre les sexes réintroduisait au sein de la fantasmagorie toute puissante du sujet rêvant le monde avec lui-même l'indispensable altérité, solidaire de la discontinuité. Ultime paradoxe: au sein de cette pièce qui a évacué le texte, le nouveau logos rêvé par l'artiste semble exclusivement pictural, mais c'est au sein de cette expression que resurgit le discontinu, indissociable de l'expression verbale, conformément à la dichotomie mise en avant par Michaux. Si l'art pictural flirte avec le continu et l'inentamable pour s'en démarquer in extremis, le commentaire sur l'art appelé par ce dispositif, ne saurait quant à lui s'effectuer que dans une langue compréhensible et partageable par une communauté de sujets. A sa manière, le dispositif voulu par l'artiste pour la présentation de son œuvre fonctionne comme arrière-texte lectoral" vis-à-vis du pseudo-texte du Cabinet logologique.

Avec l'Art Brut, poussé à ses conséquences extrêmes dans le cycle de L'Hourloupe, Dubuffet donne à voir et à concevoir certains ressorts du pôle confusionnel plus actif en régime pictural et dont son œuvre est l'une des manifestations. Symétriquement, la langue, matériau de l'expression littéraire, voit deux de ses propriétés soulignées : la discontinuité du signe, qui permet d'articuler du sens, et, moins souvent aperçue, la parenté constitutive entre langue et différence des sexes. Sans doute ce qui vient d'être exploré, grâce à Dubuffet et à son complice Michaux, à propos de la ligne, continue ou discontinue, gagnerait-il à être complété par une réflexion sur la couleur en peinture. La couleur, autre bloc inentamable par les mots. Pour savoir ce qu'est un bleu de Klein, il faut le regarder. On se contentera néanmoins de cette réponse partielle à la question de l'irréductibilité de la peinture aux mots.

Le mot dans son rapport au signifié et son équivalent visuel, la figure, arrachent l'art littéraire ou pictural au pôle confusionnel et ouvrent un espace à la lecture. Mais c'est aussi le côtoiement de la radicalité contraire qui confère à l'expérience esthétique sa puissance. L'édification du Cabinet de logologie constitue un dispositif original donnant à contempler l'envers fantasmatique des langues, dont il met en scène l'arrière-texte. Il est à noter qu'un autre grand poète du siècle, Francis Ponge, ami et admirateur, de Dubuffet, ne l'a pas suivi jusqu'à L'Hqurloupe « qu'il n'a pas compris ».12 Lenjeu de lart pour Ponge etait de (re)façonner le logos en lui imprimant sa marque ; la refondation rêvée par Dubuffet le conduit à s'approcher au maximum de sa destruction.

L'appel ultime à l'espace socialisé de la lecture trouve un prolongement dans l'adoption de la langue commune au plan de la théorie esthétique. Afin de conserver la trace de l'ébranlement recherché dans sa pratique, Dubuffet intègre au commentaire de ses travaux la contradiction ouverte d'une théorie anti-théorique. Quelque chose de ce paradoxe subsiste dans le dispositif d'accès à son œuvre picturale. La Closerie Falbala, siège d'une grande partie de cette œuvre, a été construite pour la postérité et se trouve comme soustraite aux regards. S'y rendre tient du parcours ini- 
tiatique : nous aimerions aussi que ce propos donne envie à quelques-uns d'en faire l'expérience. Mettre en paroles cette expérience déroutante, c'est alors, peut-être, convertir le (dé)plaisir de regarder en plaisir de dire

\section{Notes}

*Alain Trouvé est maître de conférences de littérature française du vingtième siècle (HDR) à l'Université de Reims, théoricien de la littérature et de la lecture. Au sein du séminaire Approches interdisciplinaires de la Lecture dont il est le co-fondateur et l'animateur depuis 2005, il a contribué à l'émergence de la notion d'arrière-texte, complément théorique de l'intertexte. Il a participé dès 2007 à la fondation du groupe LEA (Lire en Europe Aujourd'hui). Il a initié et co-organisé les premières "Rencontres Internationales de Reims : la lecture littéraire dans tous ses états" qui se sont tenues du 28 au 31 mai 2018 et ont, en synergie avec le groupe LEA, rassemblé des chercheurs de quatre continents ( Europe, Asie, Amérique, Afrique ).

' Les inconvénients de cette équivalence ont déjà été abordés par divers commentateurs. Voir par exemple à ce sujet, Michel Picard (1994), "Lecture de la perversion et perversion de la lecture ", in Comment la littérature agit-elle?, Paris, Klincksieck : 193-205.

${ }^{2}$ Voir à ce sujet notre article de 2016, «Barthes et le lexique de la critique » ( bibliographie ).

${ }^{3}$ Le terme est ensuite remplacé par scopophilie.

${ }^{4}$ L'Arrière-texte ( 2013 : Peter Lang ) dégage sous forme synthétique les grandes lignes de la réflexion collective menée à l'Université de Reims entre 2009 et 2011 dans le cadre du séminaire Approches Interdisciplinaires et Internationales de la Lecture (A2IL). Voir aussi à ce sujet notre article " Arrière-texte », in Jacques Poirier dir., Dictionnaire des mythes et concepts de la création, Reims, Epure, 2015 : 359-362 et notre essai Nouvelles Déclinaisons de l'arrière-texte (2018).

${ }^{5}$ Terme employé par Dubuffet pour caractériser ce type d'écrit.

${ }^{6}$ Ce livre fut republié en 1995 dans les écrits de l'auteur (Prospectus, III). Toutefois cette nouvelle édition ne retient de l'édition Skira que le texte, supprimant le jeu avec les images simultanément produites. Il transpose aussi la graphie manuscrite en capitales d'imprimerie. Cette double altération amoindrit sensiblement la sauvagerie de l'édition originale.

${ }^{7}$ Hypothèse confirmée par la composition de ce titre en forme de calembour et de mot-valise. On y entend à la fois « botanique » et " gerbe de nique(s) ». Le substantif «nique(s) » se lit lui-même doublement, comme moquerie et, dans un sens argotique, comme possession sexuelle.

${ }^{8}$ Cet article, après l'échec de l'Almanach, trouva place en 1953 dans le recueil La Clef des champs. Il est 
repris dans les CEuvres complètes de l'auteur, La Pléiade, III: 884-887.

${ }^{9}$ Emergences résurgences, $20^{\mathrm{e}}$ volume de la collection «Les sentiers de la création », paru en 1972, précède juste La Botte à nique, $\mathrm{n}^{\circ}$ 2I, 1973.

${ }^{10}$ Photographie personnelle prise en juillet 2020 et reproduite avec l'autorisation de Madame Sophie Webel, Directrice de la Fondation Dubuffet.

"La théorisation nouvelle de l'arrière-texte distingue et met en relation un arrière-texte auctorial, reconstitué par le lecteur, et un arrière-texte lectoral, ouvrant sur les conditions de toute nouvelle lecture.

${ }^{12}$ Nous tenons ce propos d'un échange privé avec Armande Ponge, sa fille.

\section{Bibliographie}

Aragon, Louis \& Cocteau, Jean (1957), Entretiens sur le musée de Dresde, Paris, Editions Cercle d'Art.

Barthes, Roland (1973a), Le Plaisir du texte, Paris, Le Seuil ; Ceuvres Complètes, IV [2002].

-- (1973b), « (Théorie du) Texte », Encyclopédie Universalis ; CEuvres Complètes, IV [2002].

Baumgarten, Alexandre Gottlieb, Aesthetica (1750-1758), Francfort-sur-le-Main, Paris, L'Herne, [1988].

Bonnefoy, Yves (2016), L'Écharpe rouge, Paris, Le Mercure de France.

Brun, Baptiste (2019), Jean Dubuffet et la besogne de l'Art Brut, Dijon, Les presses du réel.

Butor, Michel (1969), Les Mots dans la peinture, Genève, Skira.

Dubuffet, Jean (1967), Prospectus et tous écrits suivants (PES), Paris, Gallimard, Tomes I et II.

-- (1973), La Botte à Nique, Genève, Skira, «Les sentiers de la création »; Prospectus, III [1995].

-- (1995), Prospectus et tous écrits suivants (PES), Paris, Gallimard, Tomes III et IV.

-- (1995), Biographie au pas de course, PES, IV.

Gladieu, Marie-Madeleine, Pottier, Jean-Michel, et Trouvé, Alain (2013), L'Arrière-texte Pour repenser le littéraire, Bruxelles, Peter Lang.

Jenny, Laurent (2013), La Vie esthétique, Paris, Verdier.

-- (2020), Le Désir de voir, Paris, L’Atelier contemporain.

Luquet, Georges-Henri (1930), L'art primitif, Paris, Gaston Doin et Cie.

Michaux, Henri (1972), Emergences résurgences, Genève, Skira, «Les sentiers de la création ». 
Oury, Jean (1989), Création et schizophrénie, Paris, Galilée.

Picard, Michel (1994), «Lecture de la perversion et perversion de la lecture », in Comment la littérature agit-elle?, Paris, Klincksieck: 193-205.

Rancière, Jacques (2001), L'Inconscient esthétique, Paris, Galilée.

-- (2007), Politique de la littérature, Paris, Galilée.

Ricœur, Paul (1965), De l'interprétation, Paris, Le Seuil.

Saussure, Ferdinand de (1974), Cours de linguistique générale, Paris, Payot, [1916].

Schaeffer, Jean-Marie (1996), Les Célibataires de l'art, Paris, Gallimard.

Triolet, Elsa (1969), La Mise en mots, Genève, Skira "Les sentiers de la création ».

Trouvé, Alain (2015), "Arrière-texte ", in Jacques Poirier (dir.), Dictionnaire des mythes et concepts de la création, Reims, Epure: 359-362.

-- (2016), «Barthes et le lexique de la critique », in Exotopies de Barthes (dir. Maria de Jesus Cabral, Andrea Del Lungo et Franc Schuerewegen), Revue Carnets, ${ }^{\circ}$ spécial.

-- (2018), Nouvelles déclinaisons de l'arrière-texte, Reims, Epure.

-- (2019), «La littérature comme échange verbal différé : autour de L'Echarpe rouge (Yves Bonnefoy) », in Approches Interdisciplinaires de la Lecture, $\mathrm{n}^{\circ} 13$ (co-dir.), «Paroles de lecteurs 2 Poésie et autres genres », Reims, Epure: 15-37. 